A $\mathrm{Cublications}$ Bioorg. Med. Chem. Rep. 2:1-2 (2019) 24-31

Bioorganic \&

Medicinal

Chemistry

Reports

\title{
Synthesis and biological evaluation of substituted
} hexahydroquinoline derivatives

\author{
Gökalp Çetin 1,* and Rahime Şimşek 1 \\ ${ }^{1}$ Department of Pharmaceutical Chemistry, Faculty of Pharmacy, Hacettepe University, 06100 Ankara \\ Türkiye
}

(Received November 21, 2019; Revised December 07, 2019; Accepted December 10, 2019)

\begin{abstract}
It is known that microorganisms develop resistance to the drugs used in the treatment of diseases caused by bacteria and fungi. Therefore, the discovery of new drug molecules have great importance for the treatment of these diseases. In this study, compounds having dialkyl 4,4'-(1,4-phenylene)bis(2,6,6-trimethyl-5-oxo-1,4,5,6,7,8hexahydroquinoline-3-carboxylate) general structure which are expected to exhibit antibacterial and antifungal activity were synthesized. ${ }^{1} \mathrm{H}-\mathrm{NMR}$ and IR analyzes were performed to prove their structures. All compounds were tested against Gram (+) Staphylococcus aureus and Gram (-) Escherichia coli, Klebsiella pneumoniae, Acinetobacter baumannii, Pseudomonas aeruginosa to evaluate their in vitro antibacterial activity; Candida albicans and Cryptococcus neoformans var. grubii to evaluate in vitro antifungal activity. According to the biological activity data, the most active derivative in the series was compound $\mathbf{2}$ as an antibacterial agent and compound 7 as an antifungal agent.
\end{abstract}

Keywords: Hexahydroquinoline; Hantzsch synthesis; antibacterial; antifungal; inhibition. @2019 ACG Publication. All right reserved.

\section{Introduction}

Infection-related diseases, cardiovascular diseases and cancer are among the most important life-threatening diseases. ${ }^{1}$ It is also known that microorganisms develop resistance to drugs used in the treatment of these diseases caused by bacteria and fungi. One of the reasons for the development of resistance is the misuse of antibiotics in the treatment of bacterial and fungal infections. In addition, carbapenems and glycopeptides also cause bacterial resistance. Therefore, the treatment of infections caused by bacteria and fungi becomes difficult. As a result the discovery of new drug molecules have great importance for the treatment of these diseases. ${ }^{2,3}$

The 1,4-dihydropyridine (1,4-DHP) structure, first synthesized in 1882, took part in the treatment as a calcium channel blocker. It is a molecule that has been the subject of research for many years due to its various pharmacological activities. 1,4-DHPs exhibit antihypertensive, anticonvulsant, antitubercular, anti-inflammatory, antioxidant, antiatherosclerotic, anti-Alzheimer, antiulcer and antimicrobial activity Depending on the structure-activity relationships, modifications were made in the structure of 1,4-DHPs and many 1,4-DHP derivatives were discovered including hexahydroquinolines. It was found that the hexahydroquinolines have a wide spectrum of pharmacological effects by interacting with different molecular targets. ${ }^{4,5}$ One of the various modifications based on structureactivity on the hexahydroquinoline structure is the substitution of the phenyl ring at 4th position. This variation has been found to contribute to the activity. It has also been shown that the pharmacological activity is remained in hexahydroquinoline derivatives where the ester structure at 5 th position of the

\footnotetext{
*Corresponding author: E-Mail: gokalpcetin@ hacettepe.edu.tr
} 
1,4-DHP core is introduced into the ring system. ${ }^{6}$ In addition, modification of the ester group has been shown to play an important role for activity. Biological activity varied between hexahydroquinoline derivatives bearing different ester groups. ${ }^{7,8}$

Based on this information the compounds containing two hexadroquinoline rings of the same molecule thus carrying two equivalents of the same pharmacophore were synthesized in this study. This combination is intended to increase biological activities. In vitro antibacterial and antifungal activities of the compounds were evaluated and the results were given.

\section{Experimental}

\subsection{Chemical Material and Apparatus}

All chemicals used in this study were purchased from Aldrich (Germany). Melting points were determined with Thomas Hoover Capillary Melting Point Apparatus and were not corrected. Infrared spectra were recorded on a Perkin Elmer Spectrum $B X(v, \mathrm{~cm}-1)$. The ${ }^{1} \mathrm{H}-\mathrm{NMR}$ spectra were recorded on a Varian Mercury $400 \mathrm{MHz}$ Ultra Shield Spectrophotometer (DMSO-d6; tetramethylsilane as internal standard) spectrometer. TLC was carried out on Merck $0.2 \mathrm{~mm}$ silica gel 60 F254 analytical aluminum plates. All compounds reported in this paper were in racemic form. Other chemicals or solvents used in this study were HPLC or analytical grade.

\subsection{Chemistry}

Synthesis of compounds having a general structure of alkyl 4,4'-(1,4-phenylene)bis(2,6,6trimethyl-5-oxo-1,4,5,6,7,8-hexahydroquinoline-3-carboxylate) was taken place in a flask by refluxing 4,4-dimethyl-1,3-cyclohexadione $(2 \mathrm{mmol})$, terephthalaldehyde $(1 \mathrm{mmol})$, appropriate alkyl acetoacetate $(2 \mathrm{mmol})$ and ammonium acetate $(10 \mathrm{mmol})$ in the presence of $10 \mathrm{~mL}$ of methanol for eight hours. ${ }^{9}$ The reaction was followed by the TLC method. The reaction mixture was cooled to room temperature and poured into ice-water. The precipitated solids were filtered off. Crystallization was carried out in a methanol-water mixture for purification (Scheme 1).<smiles>[R]OC(=O)CC(C)=O</smiles><smiles>[R]OC(=O)C1=C(C)NC2=C(C(=O)C(C)(C)CC2)C1c1ccc(C2C(C(=O)O[R])=C(C)NC3=C2C(=O)C(C)(C)CC3)cc1</smiles>

Scheme 1. General synthesis of the compounds 1-7. 


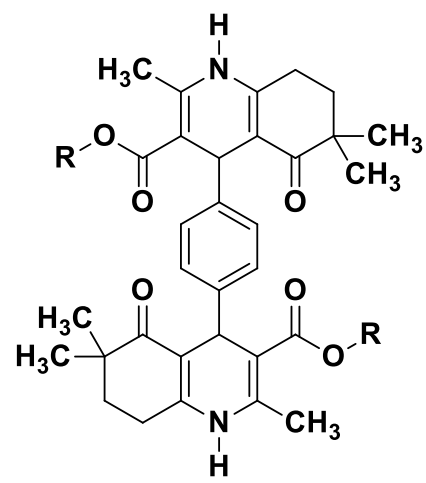

Compo
$\mathbf{1}$
$\mathbf{2}$
$\mathbf{3}$
$\mathbf{4}$
$\mathbf{5}$
$\mathbf{6}$
$\mathbf{7}$

R
Methyl
Ethyl
Isopropyl
Isobutyl
$t$-Butyl
2-(methacryloyloxy)ethyl
Benzyl

Figure 1. Structure of the compound 1-7

Methyl 4,4'-(1,4-phenylene)bis(2,6,6-trimethyl-5-oxo-1,4,5,6,7,8-hexahidroquinoline-3-carboxylate) (1): Yellowish solid, m.p. decomp $300^{\circ} \mathrm{C}$, yield: 67,9\%. FTIR $\left(\mathrm{v}, \mathrm{cm}^{-1}\right) 3303(\mathrm{~N}-\mathrm{H}$ stretching), 3082 (C-H stretching, aromatic), 2928 (C-H stretching, aliphatic), 1683 (C=O stretching, ester), 1646 (C=O stretching, ketone), 1470 (C-H, bending), 1185 (C-O stretching) and 807 (C-H, bending, p-disubstituted benzene). ${ }^{1} \mathrm{H}$ NMR $\left(\delta, \mathrm{DMSO}_{-} \mathrm{d}_{6}\right): 0.88\left(3 \mathrm{H}, \mathrm{s}, 6-\mathrm{CH}_{3}\right), 0.94\left(3 \mathrm{H}, \mathrm{s}, 6-\mathrm{CH}_{3}\right), 1.68-1.69(2 \mathrm{H}, \mathrm{m}$, quinoline $\mathrm{H}-7), 2.22\left(3 \mathrm{H}, \mathrm{s}, 2-\mathrm{CH}_{3}\right), 2.45-2.48(2 \mathrm{H}, \mathrm{m}$, quinoline $\mathrm{H}-8), 3.51\left(3 \mathrm{H}, \mathrm{s}, \mathrm{COOCH}_{3}\right), 4.78(1 \mathrm{H}, \mathrm{s}$, quinoline $\mathrm{H}-4), 6.82-6.88(2 \mathrm{H}, \mathrm{m}$, aromatic), $9.06(1 \mathrm{H}, \mathrm{s}, \mathrm{NH})$.

Ethyl4,4'-(1,4-phenylene)bis(2,6,6-trimethyl-5-oxo-1,4,5,6,7,8-hexahydroquinoline-3-carboxylate) (2): Yellowish solid, m.p: m.p. decomp $300^{\circ} \mathrm{C}$, yield: 71,1\%. IR (v, $\left.\mathrm{cm}^{-1}\right) 3277$ (N-H stretching), 3086 (C$\mathrm{H}$ stretching, aromatic), 2965 (C-H stretching, aliphatic), 1691 ( $\mathrm{C}=\mathrm{O}$ stretching, ester), 1651 (C=O stretching, ketone), 1485 (C-H, bending), 1189 (C-O stretching) and 818 (C-H, bending, p-disubstituted benzene). ${ }^{1} \mathrm{H}$ NMR $\left(\delta, \mathrm{DMSO}_{-} \mathrm{d}_{6}\right): 0.86\left(3 \mathrm{H}, \mathrm{s}, 6-\mathrm{CH}_{3}\right), 0.94\left(3 \mathrm{H}, \mathrm{s}, 6-\mathrm{CH}_{3}\right), 1.67-1.71(2 \mathrm{H}, \mathrm{m}$, quinoline $\mathrm{H}-7), 2.21\left(3 \mathrm{H}, \mathrm{s}, 2-\mathrm{CH}_{3}\right), 2.44-2.48(2 \mathrm{H}, \mathrm{m}$, quinoline $\mathrm{H}-8), 3.93\left(1 \mathrm{H}, \mathrm{dq}, \mathrm{COOCH}_{2 \mathrm{~A}} \mathrm{CH}_{3}\right), 3.99(1 \mathrm{H}$, dq, $\left.\mathrm{COOCH}_{2 \mathrm{~B}} \underline{\mathrm{CH}}_{3}\right), 4.76(1 \mathrm{H}, \mathrm{s}$, quinoline $\mathrm{H}-4), 6.89(2 \mathrm{H}, \mathrm{s}$, aromatic), $9.00(1 \mathrm{H}, \mathrm{s}, \mathrm{NH})$.

Isopropyl4,4'-(1,4-phenylene)bis(2,6,6-trimethyl-5-oxo-1,4,5,6,7,8-hexahydroquinoline-3-carboxylate) (3): Yellowish solid, m.p. decomp $300^{\circ} \mathrm{C}$, yield: 63,2\%. IR ( $\left.\mathrm{v}, \mathrm{cm}^{-1}\right) 3274$ (N-H stretching), 3093 (C$\mathrm{H}$ stretching, aromatic), 2982 (C-H stretching, aliphatic), 1690 ( $\mathrm{C}=\mathrm{O}$ stretching, ester), 1648 (C=O stretching, ketone), 1454 (C-H, bending), 1197 (C-O stretching) and $820(\mathrm{C}-\mathrm{H}$, bending, p-disubstituted benzene). ${ }^{1} \mathrm{H}$ NMR ( $\delta$, DMSO-d 6 ): $0.88\left(3 \mathrm{H} ; \mathrm{s} ; 6-\mathrm{CH}_{3}\right), 0.97\left(3 \mathrm{H} ; \mathrm{s} ; 6-\mathrm{CH}_{3}\right), 1.06\left(3 \mathrm{H}, \mathrm{d}, \mathrm{CH}\left(\mathrm{CH}_{3}\right)_{2 \mathrm{a}}\right)$, $1.16\left(3 \mathrm{H}, \mathrm{d}, \mathrm{CH}\left(\mathrm{CH}_{3}\right)_{2 \mathrm{~b}}\right), 1.68-1.72(2 \mathrm{H}, \mathrm{m}$, quinoline $\mathrm{H}-7), 2.26\left(3 \mathrm{H}, \mathrm{s}, 2-\mathrm{CH}_{3}\right), 2.49-2.51(2 \mathrm{H}, \mathrm{m}$, kinolin $\mathrm{H}-8), 4.77-4.83\left(1 \mathrm{H}, \mathrm{m}, \underline{\mathrm{CH}}\left(\mathrm{CH}_{3}\right)_{2}\right), 4.85(1 \mathrm{H}, \mathrm{s}$, kinolin $\mathrm{H}-4), 7.19-7.58$ (2H, m, aromatic), 9.06 $(1 \mathrm{H}, \mathrm{s}, \mathrm{NH})$.

Isobutyl4,4'-(1,4-phenylene)bis(2,6,6-trimethyl-5-oxo-1,4,5,6,7,8-hexahydroquinoline-3-car boxylate) (4): Yellowish solid, m.p. decomp $300^{\circ} \mathrm{C}$, yield: 59,8\%. IR $\left(v, \mathrm{~cm}^{-1}\right) 3299$ (N-H stretching), 3075 (C$\mathrm{H}$ stretching, aromatic), 2956 (C-H stretching, aliphatic), 1686 (C=O stretching, ester), $1604(\mathrm{C}=\mathrm{O}$ stretching, ketone), 1481 (C-H, bending), 1213 (C-O stretching) and 852 (C-H, bending, p-disubstituted benzene). ${ }^{1} \mathrm{H}$ NMR $(\delta$, DMSO-d 6$): 0.78\left(3 \mathrm{H}, \mathrm{d}, \mathrm{J}=8 \mathrm{~Hz}, \mathrm{CH}_{2} \mathrm{CH}\left(\mathrm{CH}_{3}\right)_{\mathrm{a}}\right), 0.79\left(3 \mathrm{H}, \mathrm{s}, 6-\mathrm{CH}_{3}\right), 0.81(3 \mathrm{H}$, d, J= $\left.4.8 \mathrm{~Hz} \mathrm{CH} \mathrm{CH}_{2}\left(\mathrm{CH}_{3}\right)_{\mathrm{b}}\right), 0.99\left(3 \mathrm{H}, \mathrm{s}, 6-\mathrm{CH}_{3}\right), 1.78-1.85\left(\mathrm{H}, \mathrm{m}, \mathrm{CH}_{2} \mathrm{CH}\left(\mathrm{CH}_{3}\right)_{2}\right), 1.98(\mathrm{H}, \mathrm{d}, \mathrm{J}=16$ $\mathrm{Hz}$, quinoline $\mathrm{H}-8 \mathrm{a}), 2.15(\mathrm{H}, \mathrm{d}, \mathrm{J}=16 \mathrm{~Hz}$, quinoline $\mathrm{H}-8 \mathrm{~b}), 2.28(\mathrm{H}, \mathrm{d}, \mathrm{J}=16 \mathrm{~Hz}$, quinoline $\mathrm{H}-6 \mathrm{a}), 2.31$ 
$\left(3 \mathrm{H}, \mathrm{s}, 2-\mathrm{CH}_{3}\right), 2.4(\mathrm{H}, \mathrm{d}, \mathrm{J}=16 \mathrm{~Hz}$, quinoline $\mathrm{H}-6 \mathrm{~b}), 3.71\left(2 \mathrm{H}, \mathrm{m}, \underline{\mathrm{CH}_{2}} \mathrm{CH}\left(\mathrm{CH}_{3}\right)_{2}\right), 4.90(1 \mathrm{H}, \mathrm{s}$, quinoline $\mathrm{H}-4), 7.21-7.58(2 \mathrm{H}, \mathrm{m}$, aromatic), $9.10(1 \mathrm{H}, \mathrm{s}, \mathrm{NH})$.

t-Butyl 14,4'-(1,4-phenylene)bis(2,6,6-trimethyl-5-oxo-1,4,5,6,7,8-hexahydroquinoline-3-car-boxylate) (5): Yellowish solid, m.p. decomp $300^{\circ} \mathrm{C}$, yield: 66,7 \%. IR ( $\left.v, \mathrm{~cm}^{-1}\right) 3284$ (N-H stretching), 3088 (C$\mathrm{H}$ stretching, aromatic), 2969 (C-H stretching, aliphatic), 1693 (C=O stretching, ester), 1602 (C=O stretching, ketone), 1487 (C-H, bending), 1160 (C-O stretching) and 821 (C-H, bending, p-disubstituted benzene). ${ }^{1} \mathrm{H}$ NMR $\left(\delta, \mathrm{DMSO}_{-} \mathrm{d}_{6}\right): 0.80\left(3 \mathrm{H}, \mathrm{s}, 6-\mathrm{CH}_{3}\right), 0.83\left(3 \mathrm{H}, \mathrm{s}, 6-\mathrm{CH}_{3}\right), 1,24\left(9 \mathrm{H}, \mathrm{s}, \mathrm{COO}\left(\mathrm{CH}_{3}\right)_{3}\right)$, 1.64-1.66 (2H, m, quinoline $\mathrm{H}-7), 2.17\left(3 \mathrm{H}, \mathrm{s}, 2-\mathrm{CH}_{3}\right), 2.40-2.43(2 \mathrm{H}, \mathrm{m}$, quinoline $\mathrm{H}-8), 4.67(1 \mathrm{H}, \mathrm{s}$, quinoline $\mathrm{H}-4), 6.89(2 \mathrm{H}, \mathrm{s}$, aromatic), $8,84(1 \mathrm{H}, \mathrm{s}, \mathrm{NH})$.

2-(methacryloyloxy) ethyl 4,4'-(1,4-phenylene)bis(2,6,6-trimethyl-5-oxo-1,4,5,6,7,8-hexahydroquinoline-3-carboxylate)(6): Yellowish solid, m.p. decomp $300^{\circ} \mathrm{C}$, yield: 63,4 \%. IR $\left(v, \mathrm{~cm}^{-1}\right) 3300(\mathrm{~N}-\mathrm{H}$ stretching), 3072 (C-H stretching, aromatic), 2926 (C-H stretching, aliphatic), 1701 (C=O stretching, ester), $1597(\mathrm{C}=\mathrm{O}$ stretching, ketone), $1483(\mathrm{C}-\mathrm{H}$, bending $), 1167(\mathrm{C}-\mathrm{O}$ stretching) and $806(\mathrm{C}-\mathrm{H}$, bending, p-disubstituted benzene) ${ }^{1} \mathrm{H}$ NMR $\left(\delta, \mathrm{DMSO}^{-} \mathrm{d}_{6}\right): 0.86\left(3 \mathrm{H}, \mathrm{s}, 6-\mathrm{CH}_{3}\right), 0.94\left(3 \mathrm{H}, \mathrm{s}, 6-\mathrm{CH}_{3}\right)$, 1.67-1.70 (2H, m, quinoline $\mathrm{H}-7), 2.25\left(3 \mathrm{H}, \mathrm{s}, 2-\mathrm{CH}_{3}\right), 2.45-2.49(2 \mathrm{H}, \mathrm{m}$, quinoline $\mathrm{H}-8), 4.83(1 \mathrm{H}, \mathrm{s}$, quinoline H-4), 4.93-5.09 (2H, m, $\left.\mathrm{COOCH}_{2} \mathrm{C}_{6} \mathrm{H}_{5}\right), 6.84(2 \mathrm{H}, \mathrm{s}$, aromatic), 7.11-7.16 $(2 \mathrm{H}, \mathrm{m}$, $\left.\mathrm{COOCH}_{2} \mathrm{C}_{6} \mathrm{H}_{5}\right), 7.23-7.27\left(3 \mathrm{H}, \mathrm{m}, \mathrm{COOCH}_{2} \mathrm{C}_{6} \mathrm{H}_{5}\right), 9.10(1 \mathrm{H}, \mathrm{s}, \mathrm{NH})$.

Benzyl 4,4'-(1,4-phenylene)bis(2,6,6-trimethyl-5-oxo-1,4,5,6,7,8-hexahydroquinoline-3-carboxylate) (7): Yellowish solid, m.p: $287-290^{\circ} \mathrm{C}$, yield: $63,4 \%$. IR ( $\left.\mathrm{v} \mathrm{cm}^{-1}\right) 3300(\mathrm{~N}-\mathrm{H}$ stretching), 3072 (C-H stretching, aromatic), 2926 (C-H stretching, aliphatic), $1701(\mathrm{C}=\mathrm{O}$ stretching, ester), 1597 ( $\mathrm{C}=\mathrm{O}$ stretching, ketone), 1483 (C-H, bending), 1167 (C-O stretching) and 806 (C-H, bending, p-disubstituted benzene). ${ }^{1} \mathrm{H}$ NMR $\left(\delta\right.$, DMSO- $\left.\mathrm{d}_{6}\right): 0.86\left(3 \mathrm{H}, \mathrm{s}, 6-\mathrm{CH}_{3}\right), 0.94\left(3 \mathrm{H}, \mathrm{s}, 6-\mathrm{CH}_{3}\right), 1.67-1.70(2 \mathrm{H}, \mathrm{m}$, quinoline $\mathrm{H}-7), 2.25\left(3 \mathrm{H}, \mathrm{s}, 2-\mathrm{CH}_{3}\right), 2.45-2.49(2 \mathrm{H}, \mathrm{m}$, quinoline $\mathrm{H}-8), 4.83(1 \mathrm{H}, \mathrm{s}$, quinoline $\mathrm{H}-4), 4.93-5.09$ $\left(2 \mathrm{H}, \mathrm{m}, \mathrm{COOCH}_{2} \mathrm{C}_{6} \mathrm{H}_{5}\right), 6.84\left(2 \mathrm{H}, \mathrm{s}\right.$, aromatic), 7.11-7.16 (2H, m, $\left.\mathrm{COOCH}_{2} \mathrm{C}_{6} \mathrm{H}_{5}\right), 7.23-7.27(3 \mathrm{H}, \mathrm{m}$, $\left.\mathrm{COOCH}_{2} \mathrm{C}_{6} \mathrm{H}_{5}\right), 9.10(1 \mathrm{H}, \mathrm{s}, \mathrm{NH})$.

\subsection{Antimicrobial Assay}

The antimicrobial activity of the all compounds were tested against Staphylococcus aureus ATCC 43300, Escherichia coli ATCC 25922, Klebsiella pneumoniae ATCC 700603, Acinetobacter baumannii ATCC 19606 and Pseudomonas aeruginosa ATCC 27853.

2.3.1. Preparation of Microbial Cultures and for Antimicrobial Activity Test of Alkyl 4,4'-(1,4phenylene)bis(2,6,6-trimethyl-5-oxo-1,4,5,6,7,8-hexahidroquinoline-3-carboxylate)

Samples were provided by the collaborator and stored frozen at $-20{ }^{\circ} \mathrm{C}$. Samples were prepared in DMSO and water to a final testing concentration of $32 \mu \mathrm{g} /{ }^{\mathrm{mL}}$ or $20 \mu \mathrm{M}$ (unless otherwise indicated in the data sheet), in 384-well, non-binding surface plate (NBS) for each bacterial/fungal strain, and in duplicate $(\mathrm{n}=2)$, and keeping the final DMSO concentration to a maximum of $1 \%$ DMSO. All the sample-preparation where done using liquid handling robots. Compounds that showed solubility issues during stock solution preparation are detailed in the data sheet.

\subsubsection{Procedure}

All bacteria were cultured in Cation-adjusted Mueller Hinton broth (CAMHB) at $37^{\circ} \mathrm{C}$ overnight. A sample of each culture was then diluted 40 -fold in fresh broth and incubated at $37^{\circ} \mathrm{C}$ for $1.5-3 \mathrm{~h}$. The resultant mid-log phase cultures were diluted $(\mathrm{CFU} / \mathrm{mL}$ measured by OD600), then added to each well of the compound containing plates, giving a cell density of 5'105 CFU/mL and a total volume of $50 \mu \mathrm{L}$. All the plates were covered and incubated at $37^{\circ} \mathrm{C}$ for $18 \mathrm{~h}$ without shaking. 


\subsubsection{Antimicrobial Data Collection}

Analysis Inhibition of bacterial growth was determined measuring absorbance at $600 \mathrm{~nm}$ (OD600), using a Tecan M1000 Pro monochromator plate reader. The percentage of growth inhibition was calculated for each well, using the negative control (media only) and positive control (bacteria without inhibitors) on the same plate as references. The significance of the inhibition values was determined by modified Z-scores, calculated using the median and MAD of the samples (no controls) on the same plate. Samples with inhibition value above $80 \%$ and Z-Score above 2.5 for either replicate $(n=2$ on different plates) were classed as actives. Samples with inhibition values between $50-80 \%$ and $\mathrm{Z}$-Score above 2.5 for either replicate ( $\mathrm{n}=2$ on different plates) were classed as partial actives.

\subsection{Antifungal Assay}

The antifungal activity of the all compounds were tested against Candida albicans ATCC 90028 and Cryptococcus neoformans var. Grubii ATCC 208821.

2.4.1. Preparation of Microbial Cultures and for Antifungal Activity Test of Alkyl 4,4'-(1,4phenylene)bis(2,6,6-trimethyl-5-oxo-1,4,5,6,7,8-hexahidroquinoline-3-carboxylate)

Samples were provided by the collaborator and stored frozen at $-20{ }^{\circ} \mathrm{C}$. Samples were prepared in DMSO and water to a final testing concentration of $32 \mu \mathrm{g} / \mathrm{mL}$ or $20 \mu \mathrm{M}$ (unless otherwise indicated in the data sheet), in 384-well, non-binding surface plate (NBS) for each bacterial/fungal strain, and in duplicate $(n=2)$, and keeping the final DMSO concentration to a maximum of $1 \%$ DMSO. All the sample-preparation where done using liquid handling robots. Compounds that showed solubility issues during stock solution preparation are detailed in the data sheet.

\subsubsection{Procedure}

Fungi strains were cultured for 3 days on Yeast Extract-Peptone Dextrose (YPD) agar at $30^{\circ} \mathrm{C}$. A yeast suspension of $1 \times 106$ to $5 \times 106 \mathrm{CFU} / \mathrm{mL}$ (as determined by OD530) was prepared from five colonies. The suspension was subsequently diluted and added to each well of the compound-containing plates giving a final cell density of fungi suspension of $2.5^{\prime} 103 \mathrm{CFU} / \mathrm{mL}$ and a total volume of $50 \mu \mathrm{L}$. All plates were covered and incubated at $35^{\circ} \mathrm{C}$ for $24 \mathrm{~h}$ without shaking.

\subsubsection{Antifungal Data Collection}

Growth inhibition of C. albicans was determined measuring absorbance at $530 \mathrm{~nm}$ (OD530), while the growth inhibition of $\mathrm{C}$. neoformans was determined measuring the difference in absorbance between 600 and $570 \mathrm{~nm}$ (OD600-570), after the addition of resazurin (0.001\% final concentration) and incubation at $35^{\circ} \mathrm{C}$ for additional $2 \mathrm{~h}$. The absorbance was measured using a Biotek Synergy HTX plate reader. The percentage of growth inhibition was calculated for each well, using the negative control (media only) and positive control (fungi without inhibitors) on the same plate. The significance of the inhibition values was determined by modified Z-scores, calculated using the median and MAD of the samples (no controls) on the same plate. Samples with inhibition value above $80 \%$ and Z-Score above 2.5 for either replicate $(\mathrm{n}=2$ on different plates) were classed as actives. Samples with inhibition values between $50-80 \%$ and Z-Score above 2.5 for either replicate ( $\mathrm{n}=2$ on different plates) were classed as partial actives. 


\section{Results and Discussion}

\subsection{Chemistry}

In this study seven compounds having a general structure of alkyl 4,4'-(1,4-phenylene)bis(2,6,6trimethyl-5-oxo-1,4,5,6,7,8-hexahydroquinoline-3-carboxylate) were synthesized by modified Hantzsch reaction. Ammonium acetate was used instead of ammonia as nitrogen source. The compounds carry alkyl chains bearing an increasing number of carbon atoms, benzyl and methacryloyloxy which include unsaturated moiety as ester functional group. Exact structures of the all compounds were elucidated by IR and ${ }^{1} \mathrm{H}-\mathrm{NMR}$ analysis. The absorption bands at around $3300 \mathrm{~cm}^{-1}$ confirmed N-H in the ring system, around 1700 confirmed ester groups and around $1600 \mathrm{~cm}^{-1}$ confirmed ketone groups. As expected ${ }^{1} \mathrm{H}-\mathrm{NMR}$ spectra of the compounds displayed singlet signals belonging to $-\mathrm{CH}_{3}$ groups at $0.9 \mathrm{ppm}$, multiplet signals belonging to aromatic protons at $7.0 \mathrm{ppm}$ and singlet signals belonging to $-\mathrm{NH}$ groups at $9 \mathrm{ppm}$. The peaks belonging to other protons were seen in expected values of the compounds' spectras.

\subsection{Antimicrobial and Antifungal Activity}

The in vitro antimicrobial activity of the all compounds were tested against Staphylococcus aureus ATCC 43300, Escherichia coli ATCC 25922, Klebsiella pneumoniae ATCC 700603, Acinetobacter baumannii ATCC 19606 and Pseudomonas aeruginosa ATCC 27853. The percentage of growth inhibition was calculated for each well, using the negative control (media only) and positive control (bacteria without inhibitors) on the same plate as references. The significance of the inhibition values was determined by modified Z-scores, calculated using the median and MAD of the samples (no controls) on the same plate.

The antifungal activities of the all compounds were tested against Candida albicans ATCC 90028 and Cryptococcus neoformans var. Grubii ATCC 208821. The percentage of growth inhibition was calculated for each well, using the negative control (media only) and positive control (fungi without inhibitors) on the same plate. The significance of the inhibition values was determined by modified Zscores, calculated using the median and MAD of the samples (no controls) on the same plate (Table 1 and Table 2).

Table 1. General Information of Microorganisms

\begin{tabular}{|c|c|c|c|c|c|c|}
\hline Abbreviation & Code & Name & Description & Strain & Organsim & Type \\
\hline $\mathrm{Sa}$ & GP_020 & Staphylococcus aureus & MRSA & ATCC 43300 & Bacteria & G+ve \\
\hline $\mathrm{Ec}$ & GN_001 & Escherichia coli & FDA control & ATCC 25922 & Bacteria & G-ve \\
\hline $\mathrm{Kp}$ & GN_003 & Klebsiella pneumoniae & MDR & $\begin{array}{l}\text { ATCC } \\
700603\end{array}$ & Bacteria & G-ve \\
\hline $\mathrm{Ab}$ & GN_034 & Acinetobacter baumannii & Type strain & ATCC 19606 & Bacteria & G-ve \\
\hline $\mathrm{Pa}$ & GN_042 & Pseudomonas aeruginosa & Type strain & ATCC 27853 & Bacteria & G-ve \\
\hline $\mathrm{Ca}$ & FG_001 & Candida albicans & $\begin{array}{l}\text { CLSI } \\
\text { reference }\end{array}$ & ATCC 90028 & Fungi & Yeast \\
\hline $\mathrm{Cn}$ & FG_002 & $\begin{array}{c}\text { Cryptococcus neoformans } \\
\text { var. grubii }\end{array}$ & Type strain & $\begin{array}{c}\text { H99; ATCC } \\
208821\end{array}$ & Fungi & Yeast \\
\hline
\end{tabular}


Table 2. Biological activity results of all compounds

\begin{tabular}{|c|c|c|c|c|}
\hline Compound & Organism & Inhibition & Z-score & $\begin{array}{c}\text { Concentration } \\
(\mu \mathrm{g} / \mathrm{mL})\end{array}$ \\
\hline 1 & Staphylococcus aureus & 14.60 & -0.55 & 32.00 \\
\hline 1 & Escherichia coli & -3.07 & 0.09 & 32.00 \\
\hline 1 & Klebsiella pneumoniae & 10.51 & -0.24 & 32.00 \\
\hline 1 & Acinetobacter baumannii & 5.18 & 0.44 & 32.00 \\
\hline 1 & Pseudomonas aeruginosa & 3.82 & 1.04 & 32.00 \\
\hline 1 & Candida albicans & 4.71 & -1.21 & 32.00 \\
\hline 1 & Cryptococcus neoformans & -27.93 & -1.65 & 32.00 \\
\hline 2 & Staphylococcus aureus & 22.49 & -0.90 & 32.00 \\
\hline 2 & Escherichia coli & -0.10 & -0.39 & 32.00 \\
\hline 2 & Klebsiella pneumoniae & 21.63 & -1.00 & 32.00 \\
\hline 2 & Acinetobacter baumannii & 28.63 & -1.38 & 32.00 \\
\hline 2 & Pseudomonas aeruginosa & 5.61 & 0.80 & 32.00 \\
\hline 2 & Candida albicans & -3.95 & 1.31 & 32.00 \\
\hline 2 & Cryptococcus neoformans & -65.54 & -0.75 & 32.00 \\
\hline 3 & Staphylococcus aureus & N/D & $\mathrm{N} / \mathrm{D}$ & 32.00 \\
\hline 3 & Escherichia coli & N/D & N/D & 32.00 \\
\hline 3 & Klebsiella pneumoniae & N/D & N/D & 32.00 \\
\hline 3 & Acinetobacter baumannii & N/D & N/D & 32.00 \\
\hline 3 & Pseudomonas aeruginosa & N/D & N/D & 32.00 \\
\hline 3 & Candida albicans & N/D & N/D & 32.00 \\
\hline 3 & Cryptococcus neoformans & N/D & N/D & 32.00 \\
\hline 4 & Staphylococcus aureus & N/D & N/D & 32.00 \\
\hline 4 & Escherichia coli & N/D & N/D & 32.00 \\
\hline 4 & Klebsiella pneumoniae & N/D & N/D & 32.00 \\
\hline 4 & Acinetobacter baumannii & N/D & N/D & 32.00 \\
\hline 4 & Pseudomonas aeruginosa & N/D & N/D & 32.00 \\
\hline 4 & Candida albicans & N/D & N/D & 32.00 \\
\hline 4 & Cryptococcus neoformans & N/D & N/D & 32.00 \\
\hline 5 & Staphylococcus aureus & 13.41 & -0.18 & 32.00 \\
\hline 5 & Escherichia coli & -9.31 & 1.77 & 32.00 \\
\hline 5 & Klebsiella pneumoniae & 0.32 & 0.73 & 32.00 \\
\hline 5 & Acinetobacter baumannii & -5.14 & 1.30 & 32.00 \\
\hline 5 & Pseudomonas aeruginosa & 20.80 & -1.27 & 32.00 \\
\hline 5 & Candida albicans & 0.64 & 0.18 & 32.00 \\
\hline 5 & Cryptococcus neoformans & -100.41 & 0.47 & 32.00 \\
\hline 6 & Staphylococcus aureus & 18.72 & -0.89 & 32.00 \\
\hline 6 & Escherichia coli & 0.95 & -0.63 & 32.00 \\
\hline 6 & Klebsiella pneumoniae & 17.66 & -0.68 & 32.00 \\
\hline 6 & Acinetobacter baumannii & 21.74 & -0.95 & 32.00 \\
\hline 6 & Pseudomonas aeruginosa & 15.90 & -0.60 & 32.00 \\
\hline 6 & Candida albicans & 15.23 & -4.16 & 32.00 \\
\hline 6 & Cryptococcus neoformans & -91.25 & 0.19 & 32.00 \\
\hline 7 & Staphylococcus aureus & 11.06 & 0.00 & 32.00 \\
\hline 7 & Escherichia coli & -2.34 & 0.13 & 32.00 \\
\hline 7 & Klebsiella pneumoniae & 3.12 & 0.50 & 32.00 \\
\hline 7 & Acinetobacter baumannii & 0.04 & 0.39 & 32.00 \\
\hline 7 & Pseudomonas aeruginosa & 10.79 & 0.09 & 32.00 \\
\hline 7 & Candida albicans & 18.00 & -4.99 & 32.00 \\
\hline 7 & Cryptococcus neoformans & -93.19 & 0.25 & 32.00 \\
\hline
\end{tabular}

According to biological activity results all inhibition values are below $50 \%$ and Z-Scores are below 2.5. 


\section{Conclusion}

We synthesized seven substituted hexahydroquinoline derivatives with modified Hantzsch reaction. The in vitro antibacterial and antifungal activity were evaluated with Gram-positive, Gramnegative organisms and fungi. Although no significant antimicrobial activity was observed in the compounds, the compound 2 had the highest inhibition value against Gram-positive bacteria and Gramnegative bacterias. The compound with the highest inhibition value against fungi is compound 7 . The antimicrobial activity of the compound 3 and 4 could not determined.

\section{Acknowledgements}

This study was supported by the Scientific Research Unit of Hacettepe University (Project no: THD 2017-13452)

The antimicrobial screening performed by CO-ADD (The Community for Antimicrobial Drug Discovery) was funded by the Welcome Trust (UK) and the University of Queensland (Australia).

\section{ORCID}

Gökalp Çetin: 0000-0001-9820-4690

Rahime Şimşek: 0000-0002-8467-6336

\section{References}

[1] Koene, R. J.; Prizment, A. E.; Blaes, A.; Konety, S. H. Shared risk factors in cardiovascular disease and cancer. Circulation 2016, 133(11), 1104-1114.

[2] Laskowska, K. N.; Gluszek, K.; Piktel, E.; Pajuste, K.; Bonita, D.; Krol, G.; Wilczewska, A.; Ajanmey, P.; Plotniece, A.; Bucki, R. Bactericidal and immunomodulatory properties of magnetic nanoparticles functionalized by 1,4-dihydropyridines. Int. J. Nanomed. 2018, 13, 3411-3424.

[3] Olejnikova, P.; Svorc, L.; Olsovska, D.; Panakova, A.; Vihonska, Z.; Kovaryova, K.; Marchalin, S. Antimicrobial activity of novel C2-substituted 1,4-dihydropyridine analogues. Sci. Pharm. 2014, 82, 221232.

[4] Gündüz, M. G.; Ragno, G.; Şimşek, R.; De Luca, M.; Şafak, C.; Grande, F.; El-Khouly, A.; İşli, F.; Yıldırım, Ş.; Fincan, G.S.Ö.; Ioele, G. Synthesis and photodegradation studies of analogues of muscle relaxant 1,4-dihydropyridine compounds. Acta Pharm. 2017, 341-355.

[5] Schade, D.; Lanier M.; Willems, E.; Okolotowicz, K.; Bushway, P.; Wahlquist, C.; Gilley, C.; Mercola, M.; Cashman, J. R. Synthesis and SAR of b-annulated 1,4-dihydropyridines define cardiomyogenic compounds as novel inhibitors of TGF $\beta$ signaling. J. Med. Chem. 2012, 55, 9946-9957.

[6] Carosati, E.; Ioan, P.; Micucci, M.; Broccatelli, F.; Cruciani, G.; Zhorov, B. S.; Chiarini, A.; Budriesi, R. 1,4-Didydropyridine scaffold in medicinal chemistry, the story so far and perspectives (Part 2): Action in other targets and antitargets. Current Med. Chem. 2012, 19(25), 4306-4323.

[7] Bülbül, B.; Öztürk, G. S.; Vural, M.; Şimşek, R.; Sarığlu, Y.; Linden, A.; Ülgen, M.; Şafak, C. Condensed 1,4-dihydropyridines with various esters and their calcium channel antagonist activities. Eur. J. Med. Chem. 2009, 44, 2052-2058.

[8] Bladen, C.; Gündüz, M. G.; Şimşek, R.; Şafak, C.ç; Zamponi, G. W. Synthesis and evaluation of 1,4dihydropyridine derivatives with calcium channel blocking activity. Pflugers Arch.-Eur. J. Physiol. 2014, 466, 1355-1363.

[9] Ranjbar, S.; Edrak, N.; Firuzi, O.; Khoshneviszadeh, M.; Miri, R. 5-Oxo-hexahydroquinoline: An attractive scaffold with diverse biological activities. Mol. Divers. 2019, 23, 471-508.

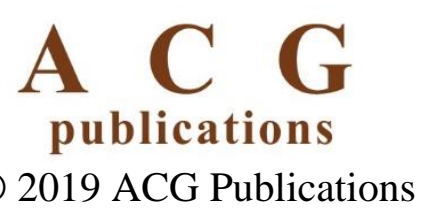

